PROCEEDINGS OF THE

AMERICAN MATHEMATICAL SOCIETY

Volume 127, Number 6, Pages 1705-1709

S 0002-9939(99)04667-5

Article electronically published on February 11, 1999

\title{
ON SYMMETRY OF DISCRETE POLYNOMIAL HYPERGROUPS
}

\author{
MARC-OLIVIER GEBUHRER AND RYSZARD SZWARC
}

(Communicated by J. Marshall Ash)

\begin{abstract}
Let $H$ be a discrete polynomial hypergoup on $\mathbb{N}$ with Plancherel measure $\mu$. If the hypergroup $H$ is symmetric, the set of characters $\widehat{H}$ can be identified with a compact subset of the real line which contains the support of $\mu$. We show that the lower and upper bounds of $\widehat{H}$ and $\operatorname{supp} \mu$ coincide. In particular, the trivial character belongs to the support of the Plancherel measure.
\end{abstract}

\section{INTRODUCTION}

A locally compact group $G$ is called amenable if it admits an invariant mean on bounded functions on $G$. One of the known characterizations of amenability is that every irreducible representation of $G$ is weakly contained in the regular representation of $G$. Actually, it suffices that the trivial representation is weakly contained in the regular representation (see [1, 4]).

Similar properties can be studied for commutative hypergroups. The set of all characters of the hypergroup $H$ can be viewed as the set of all irreducible representations in the group case. The characters which belong to the support of the Plancherel measure of the hypergroup can be viewed as the set of all irreducible representations which are weakly contained in the regular representation. We are going to study the relation between the support of the Plancherel measure $\mu$ and the set of all characters $\widehat{H}$. We will focus on special hypergroups called discrete polynomial hypergroups, described in detail in the next paragraph. The main result of this paper states that if a discrete polynomial hypergroup is symmetric, i.e. the set of all characters $\widehat{H}$ is a subset of $\mathbb{R}$, then the trivial character belongs to the support of the Plancherel measure $\mu$. Moreover we show that the lower and upper bounds of $\operatorname{supp} \mu$ and $\widehat{H}$ coincide. In particular, if $\operatorname{supp}(\mu)$ is an interval and $\widehat{H} \subset \mathbb{R}$, then $\operatorname{supp}(\mu)=\widehat{H}$.

We think that the results in this paper combined with those obtained in [7] suggest that the following conjecture should be true.

Assume that the trivial character 1 belongs to the support

of the Plancherel measure $\operatorname{supp}(\mu)$. Then $\widehat{H}=\operatorname{supp}(\mu)$.

Received by the editors January 21, 1997 and, in revised form, September 9, 1997.

1991 Mathematics Subject Classification. Primary 43A62, 42C05.

Key words and phrases. Hypergroup, symmetry, orthogonal polynomials.

This work has been partially supported by KBN (Poland) under grant 2 P03A 03009 . The work has been done while the second author was visiting IRMA, University Louis Pasteur in February 1994.

(C)1999 American Mathematical Society 
Now we turn to a detailed description of discrete polynomial hypergroups. Let $\left\{p_{n}\right\}_{n=0}^{\infty}$ be a system of orthonormal polynomials relative to a probability $\mu$ on the real line. Since the polynomials $p_{n}$ are determined up to a nonzero multiple of absolute value 1 , we may assume that they have positive leading coefficients. The polynomials $p_{n}$ satisfy a three term recurrence relation of the form

$$
x p_{n}(x)=\lambda_{n+1} p_{n+1}(x)+\beta_{n} p_{n}(x)+\lambda_{n} p_{n-1}(x) .
$$

We also assume that support of $\mu$ is bounded and that 1 is an upper bound of this set. Since the orthogonal polynomials cannot change sign in the interval $[1, \infty)$ and they tend to infinity, the values $p_{n}(1)$ are all positive. Introduce the renormalized polynomials $R_{n}(x)$ as

$$
R_{n}(x)=\frac{p_{n}(x)}{p_{n}(1)}
$$

The polynomials $R_{n}(x)$ yield a hypergroup structure if the coefficients in the product linearization formula

$$
R_{m}(x) R_{n}(x)=\sum_{k=|n-m|}^{n+m} g(m, n, k) R_{k}(x)
$$

are nonnegative for any $n$ and $m$.

We define the convolution $*$ of two measures $\nu_{1}$ and $\nu_{2}$ on $\mathbb{N}$ by means of the coefficients $g(n, m, k)$ according to

$$
\left(\nu_{1} * \nu_{2}\right)(k)=\sum_{m, n=0}^{\infty} g(m, n, k) \nu_{1}(m) \nu_{2}(m) .
$$

With this operation $M(\mathbb{N})$, the space of complex valued finite measures on $\mathbb{N}$ becomes a Banach algebra. The couple $H=(M(\mathbb{N}), *)$ is called a discrete polynomial hypergoup. The function

$$
\omega(n)=\left(\int_{-\infty}^{\infty} R_{n}(x)^{2} d \mu(x)\right)^{-1}
$$

is called the Haar measure because

$$
\nu * \omega=c \omega \quad c=\nu(\mathbb{N})
$$

for any finite measure $\nu$. On the other hand, $\mu$ is called the Plancherel measure because one has

$$
\sum_{n=0}^{\infty}|\widehat{f}(n)|^{2} \omega_{n}=\int_{-\infty}^{\infty}|f(x)|^{2} d \mu(x),
$$

where $\widehat{f}(n)=\int f(x) R_{n}(x) d \mu(x)$.

The maximal ideal space of the algebra $H=(M(\mathbb{N}), *)$ can be identified with the set

$$
\begin{aligned}
\widehat{H} & =\left\{z \in \mathbb{C}: \sup _{n}\left|R_{n}(z)\right| \leq 1\right\} \\
& =\left\{z \in \mathbb{C}:\left|p_{n}(z)\right| \leq p_{n}(1), n \in \mathbb{N}\right\} .
\end{aligned}
$$

The characters corresponding to $z \in \widehat{H}$ are defined by

$$
M(\mathbb{N}) \ni\left\{a_{n}\right\}_{0}^{\infty} \mapsto \sum_{n=0}^{\infty} a(n) R_{n}(z) .
$$


In this way the point $z=1$ corresponds to the trivial character. The number $z=1$ is the least upper bound of the real part of $\widehat{H}$ because $R_{n}(x)>R_{n}(1)=1$, for $x>1$.

In this work we investigate the relation between $\widehat{H}$ and $\operatorname{supp}(\mu)$. We always have $\operatorname{supp}(\mu) \subset \widehat{H}$ (see [3, Theorem 7.3C, p. 41] and [6, Theorem 1]). If the numbers $\omega(n)$ do not grow too fast, then the sets $\widehat{H}$ and $\operatorname{supp}(\mu)$ coincide (see $[2,7,8]$ ). In particular, the algebra $(M(\mathbb{N}), *)$ is symmetric; i.e. its maximal ideal space is a subset of the real line. The main result of this paper gives the partial converse to this statement.

\section{The RESUlts}

Lemma. Let orthonormal polynomials $\left\{p_{n}\right\}_{0}^{\infty}$ satisfy (1). Let $\mathrm{m}=\inf \operatorname{supp}(\mu)$ and $\mathrm{M}=\sup \operatorname{supp}(\mu)$. Then

$$
\mathrm{m}<\beta_{n}<\mathrm{M},
$$

if $\mu$ is not concentrated in one point.

Proof. Multiplying both sides of (1) by $p_{n}$ and integrating with respect to $\mu$ give

$$
\beta_{n}=\int_{-\infty}^{\infty} x p_{n}^{2}(x) d \mu(x)
$$

Thus the lemma follows.

Proposition. Assume the orthonormal polynomials $\left\{p_{n}\right\}_{0}^{\infty}$ satisfy (1). Let $x>$ $y>M$ or $x<y<m$. Then

$$
\frac{p_{n}(x)}{p_{n}(y)} \geq \prod_{i=0}^{n-1} \frac{x-\beta_{i}}{y-\beta_{i}}
$$

Proof. Let $x>y>M$. The polynomials $p_{n}$ take positive values in $x$ and $y$. By the Christoffel-Darboux formula we have

$$
p_{n}(x) p_{n-1}(y)-p_{n-1}(x) p_{n}(y)=\frac{x-y}{\lambda_{n}} \sum_{k=0}^{n-1} p_{k}(x) p_{k}(y) .
$$

Hence

$$
p_{n}(x) p_{n-1}(y)-p_{n-1}(x) p_{n}(y) \geq \frac{x-y}{\lambda_{n}} p_{n-1}(x) p_{n-1}(y) .
$$

Dividing both sides by $p_{n-1}(y) p_{n}(y)$ and using the fact that

$$
\lambda_{n} p_{n}(y) \leq\left(y-\beta_{n-1}\right) p_{n-1}(y)
$$

implies

This yields

$$
\frac{p_{n}(x)}{p_{n}(y)}-\frac{p_{n-1}(x)}{p_{n-1}(y)} \geq\left(\frac{x-y}{y-\beta_{n-1}}\right) \frac{p_{n-1}(x)}{p_{n-1}(y)} .
$$

$$
\frac{p_{n}(x)}{p_{n}(y)} \geq\left(\frac{x-\beta_{n-1}}{y-\beta_{n-1}}\right) \frac{p_{n-1}(x)}{p_{n-1}(y)}
$$

The case $x<y<m$ can be proved similarly, by making obvious modifications using the fact that $(-1)^{n} p_{n}(x)>0$ for $x<m$.

Theorem. Assume the hypergroup associated with $\left\{p_{n}\right\}$ is symmetric; i.e. the maximal ideal space $\widehat{H}$ is a subset of the real line. Then the set $\widehat{H}$ is contained in the closed interval $[m, M]$, where $m=\inf \operatorname{supp}(\mu)$ and $M=\sup \operatorname{supp}(\mu)$. 
Proof. By (4) we have $\sup \widehat{H}=1$. Let $m_{0}=\inf \widehat{H}$. Assume the statement is not true. Then either $M<1$ or $m_{0}<m$. We will assume $M<1$ (the case $m_{0}<m$ can be dealt with similarly). In view of the lemma we have

$$
\frac{p_{n}(x)}{p_{n}(y)} \geq\left(\frac{x-m}{y-m}\right)^{n}, \quad x>y>M .
$$

Let $M^{\prime}=\frac{1}{2}(M+1)$. Then we have

$$
p_{n}(y) \leq p_{n}(1)\left(\frac{M^{\prime}-m}{1-m}\right)^{n}, \quad \text { for } M \leq y \leq M^{\prime} .
$$

Let $z$ belong to the ellipse with foci at $M$ and $M^{\prime}$, and half-axes $a$ and $b$, where

$$
a^{2}-b^{2}=\frac{1}{4}\left(M^{\prime}-M\right)^{2} .
$$

By [5, Problem III.6.2.270] we have

$$
\left|p_{n}(z)\right| \leq p_{n}(1)\left(\frac{M^{\prime}-m}{1-m}\right)^{n}\left(\frac{2(a+b)}{M^{\prime}-M}\right)^{n} .
$$

Since $M^{\prime}-m<1-m$ we can find an ellipse $E$, with half-axes $a$ and $b$ such that

$$
\frac{2(a+b)}{M^{\prime}-M}=\frac{1-m}{M^{\prime}-m}
$$

Hence, if $z$ belongs to $E$, it satisfies

$$
\left|p_{n}(z)\right| \leq p_{n}(1)
$$

This implies that $z \in \widehat{H}$; i.e. $E \subset \widehat{H}$. Thus $\widehat{H}$ is not contained in the real line.

Corollary. Let the hypergroup $H$ associated with polynomials $\left\{p_{n}\right\}$ be symmetric and the support of the orthogonality measure $\mu$ of $\left\{p_{n}\right\}$ coincide with a closed interval $[a, b]$. Then the maximal ideal space $\widehat{H}$ is equal to $[a, b]$.

Examples. Let

$$
2 x p_{n}(x)=p_{n+1}(x)+p_{n-1}(x), \quad n \geq 1,
$$

and $p_{0}(x)=1, p_{1}(x)=x$. The polynomials satisfying (6) are called the Chebyshev polynomials and are given explicitly by the formula

$$
p_{n}(\cos t)=\cos n t .
$$

They are orthogonal on $(-1,1)$ with respect to the weight $\left(1-x^{2}\right)^{-1 / 2} d x$. We will define hypergroup $H_{a}$ by normalizing $p_{n}$ at $x=a$ for $a \geq 1$. The Plancherel measure does not depend on $a$ and

$$
d \mu_{a}(x)=\left(1-x^{2}\right)_{+}^{-1 / 2} d x .
$$

Hence supp $\mu_{a}=[-1,1]$. The set of characters can be identified with the ellipse

$$
\widehat{H_{a}}=\{z \in \mathbb{C}|| z-1|+| z+1 \mid \leq 2 a\} .
$$

For $a=1$ we get $\widehat{H_{1}}=[-1,1]$, i.e. $\widehat{H_{1}}=\operatorname{supp} \mu_{1}$. For $a>1$ the hypergroup $H_{a}$ is nonsymmetric, hence $\widehat{H_{a}} \neq \operatorname{supp} \mu_{a}$. Also in the second case, the trivial character is isolated from the support of the Plancherel measure.

We do not know of any example of a symmetric discrete polynomial hypergroup for which the support of the Plancherel measure is different from $\widehat{H}$. We conjectured in the introduction that such hypergroups do not exist. 


\section{REFERENCES}

[1] A. Hulanicki, Groups whose regular representation weakly contains all unitary representations, Studia Math. 24 (1964), 37-59. MR 33:225

[2] - On positive functionals on a group algebra multiplicative on a subalgebra, Studia Math. 37 (1971), 163-171. MR 46:9645

[3] R. I. Jewett, Spaces with an abstract convolution of measures, Adv. in Math. 18 (1975), 1-101. MR 52:14840

[4] A. L. T. Paterson, Amenability, Mathematical Surveys and Monographs 29, Amer. Math. Soc., Providence, Rhode Island, 1988. MR 90e:43001

[5] G. Polya and G. Szegö, Aufgaben und Lehrsätze aus der Analysis, Springer Verlag, New York 1964. MR 30:1219a

[6] R. Szwarc, Convolution structures associated with orthogonal polynomials, J. Math. Anal. Appl. 170 (1992), 158-170. MR 93j:42015

[7] _ A lower bound for orthogonal polynomials with an application to polynomial hypergroups, J. Approx. Theory 81 (1995), 145-150. MR 96j:42015

[8] M. Vogel, Spectral synthesis on algebras of orthogonal polynomials series, Math. Z. 194 (1987), 99-116. MR 88b:43004

Institut de Recherche Mathématique Avancée, Université Louis Pasteur et C.N.R.S., 7, rue René Descartes, 67084 Strasbourg Cedex, France

Institute of Mathematics, WrocŁaw University, Pl. Grunwaldzki 2/4, 50-384 WrocŁaW, Poland 